\title{
Working-age adults with diabetes experience greater susceptibility to seasonal influenza: a population-based cohort study
}

\author{
Darren Lau • Dean T. Eurich • Sumit R. Majumdar • \\ Alan Katz • Jeffrey A. Johnson
}

Received: 18 June 2013 / Accepted: 9 December 2013 / Published online: 6 February 2014

(C) Springer-Verlag Berlin Heidelberg 2014

\begin{abstract}
Aims/hypothesis The aim of this work was to compare the incidence of illness attributable to influenza in working-age adults (age $<65$ years) with and without diabetes.

Methods We performed a cohort study using administrative data from Manitoba, Canada, between 2000 and 2008. All working-age adults with diabetes were identified and matched with up to two non-diabetic controls. We analysed the rates of influenza-like illness physician visits and hospitalisations, pneumonia and influenza hospitalisations, and all-cause hospitalisations. Multivariable regressions were used to estimate the influenza-attributable rate of each outcome.

Results We included 745,777 person-years of follow-up among 166,715 subjects. The median age was $50-51$ years and $48-49 \%$ were women; adults with diabetes had more comorbidities and were more likely to be vaccinated for influenza than those without diabetes. Compared with similar adults without diabetes, those with diabetes had a $6 \%$ greater (RR 1.06, 95\% CI 1.02, 1.10; absolute risk difference 6 per 1,000 adults per year) increase in all-cause hospitalisations associated with influenza, representing a total of 54 additional
\end{abstract}

Electronic supplementary material The online version of this article (doi:10.1007/s00125-013-3158-8) contains peer-reviewed but unedited supplementary material, which is available to authorised users.

D. Lau • D. T. Eurich · J. A. Johnson $(\bowtie)$

Department of Public Health Sciences, School of Public Health,

University of Alberta, 2-040G Li Ka Shing Center for Health

Research Innovation, 8602112 Street, Edmonton, AB,

Canada T6G 2E1

e-mail: jeff.johnson@ualberta.ca

S. R. Majumdar

Department of Medicine, Faculty of Medicine and Dentistry,

University of Alberta, Edmonton, AB, Canada

A. Katz

Department of Community Health Sciences, Faculty of Medicine, University of Manitoba, Winnipeg, MB, Canada hospitalisations. There were no differences in the influenzaattributable rates of influenza-like illness $(p=0.06)$ or pneumonia and influenza $(p=0.11)$.

Conclusions/interpretation Guidelines calling for influenza vaccinations in diabetic, in addition to elderly, adults implicitly single out working-age adults with diabetes. The evidence supporting such guidelines has hitherto been scant. We found that working-age adults with diabetes appear more susceptible to serious influenza-attributable illness. These findings represent the strongest available evidence for targeting diabetes as an indication for influenza vaccination, irrespective of age.

Keywords Diabetes $\cdot$ Epidemiology $\cdot$ Influenza $\cdot$ Influenza vaccination

$\begin{array}{ll}\text { Abbreviations } \\ \text { ADG } & \text { Aggregate diagnostic group } \\ \text { ALL } & \text { All-cause hospitalisation } \\ \text { ILI } & \begin{array}{l}\text { Influenza-like illness (physician visit and } \\ \text { hospitalisation) }\end{array} \\ \text { pH1N1 } & \text { Pandemic H1N1 } \\ \text { PI } & \text { Pneumonia and influenza (hospitalisation) } \\ \text { RSV } & \text { Respiratory syncytial virus } \\ \text { SES } & \text { Socioeconomic status }\end{array}$

Introduction

Influenza is a common viral illness [1] responsible for substantial morbidity and mortality [2-5]. Clinical practice guidelines promulgated by diabetes mellitus care organisations (e.g. American Diabetes Association, Canadian Diabetes Association), as well as national vaccination authorities in such countries as Canada and the UK, recommend routine vaccinations against seasonal influenza in all adults with 
diabetes [6-9]. While guidelines in the USA have recently begun calling for universal vaccination of all adults, they continue to prioritise those with diabetes, particularly when resources are scarce. Since recommendations already exist for vaccination in all elderly adults (age $>65$ years), the incremental effect of guidelines calling for vaccinations in diabetic adults is to add working-age adults (age $\geq 18$ and $<65$ years) with diabetes as a high-risk group relative to those without diabetes [6-9].

Such guidelines may be justified if working-age adults with diabetes experience greater susceptibility or severity of influenza relative to similar non-diabetic adults. However, the evidence for this premise is limited $[6,7,10]$. To our knowledge, only three studies have compared the burden of seasonal influenza in adults with and without diabetes. These studies have several limitations, including potential bias from the use of hospital-based comparison groups [11], lack of adjustment for comorbidities and vaccination status [11-13] and inadequate adjustment for seasonality [12]. More recently, studies of pandemic H1N1 (pH1N1) influenza have drawn varying conclusions regarding the extent to which diabetes increases the risk of severe outcomes. Given the limitations of the present literature, we performed a population-based cohort study to test the hypothesis that working-age adults with diabetes experience greater incidence of illness due to seasonal influenza.

\section{Methods}

Setting Our study was performed using administrative data from Manitoba, Canada. Nearly all ( $>99 \%)$ residents of Manitoba have provincially funded healthcare benefits under Manitoba's system of universal health insurance [14]. The databases of Manitoba Health capture the following information: services, diagnoses and interventions provided to patients during hospital admissions and physician visits; demographics; pharmaceuticals dispensed in the community at the point of sale and vaccinations provided to Manitoba residents (www.umanitoba.ca/faculties/medicine/units/community health_sciences/departmental_units/mchp/resources/ repository/health admin.html). We have recently used these databases in an observational study of the effectiveness of influenza vaccination in working-age adults with diabetes [15]. The present study was approved by the Institutional Review Board of the University of Alberta (Pro00026010) and by the Health Information and Privacy Committee of Manitoba (HIPC 2011/2012-16).

Subjects We identified all working-age adults with diabetes mellitus, from 1 July 2000 to 30 June 2008. Adults with both prevalent and incident diabetes were selected, using a wellvalidated claims-based definition of diabetes. Adults were defined as having diabetes after two ambulatory physician claims or one hospital discharge for diabetes mellitus (ICD-9 code 250 [www.icd9data.com/2007/Volume1/240-279/250259/250/default.htm] or ICD-10 codes E10-E11 [www.who. int/classifications/icd/en/]) [16]. A non-diabetic control group was composed by matching diabetic adults with up to two non-diabetic adults by age (i.e. \pm 1 year), sex and health region. A priori calculations suggested that a 2:1 match would provide sufficient power for the analyses planned. Non-diabetic subjects were followed from the date of their diabetic counterpart's diagnosis of diabetes.

We divided calendar time into years from 1 July to 30 June [12]. Influenza season was defined as a continuous period between the first and last occurrences of at least two consecutive weeks with two or more isolates positive for influenza, according to provincial surveillance data [17-19].

Outcomes and follow-up We followed all subjects, until 30 June 2008, for any occurrences of three outcomes, based on ICD diagnostic codes: physician visit or hospitalisation for influenza-like illness (ILI), hospitalisation for pneumonia and influenza (PI) and all-cause hospitalisation (ALL). Influenzalike illness is usually defined as a febrile cough with additional symptoms of systemic involvement [20]. However, ICD codes do not contain a specific entry for ILI, as defined clinically. We therefore adopted an administrative case definition of ILI, which consisted of a broad bundle of diagnoses, including bronchitis, pneumonia, cold, cough and exacerbations of chronic obstructive pulmonary disease (see electronic supplementary material [ESM] Tables 1 and 2). This case definition, determined in a pilot study of six emergency departments, is similar to those of other studies identifying diagnoses correlated with influenza activity [21, 22]. ILI was chosen to represent the common manifestations of influenza, which include PI hospitalisations. PI was also examined as a separate outcome to depict more serious and specific respiratory sequelae. ALL is non-specific, but was included to indicate the overall burden of influenza on serious morbidity, since influenza is thought to contribute to a significant range of non-respiratory outcomes including myocardial infarction and diabetic ketoacidosis [2-5]. Subjects were able to contribute multiple outcomes.

Measurement approach-influenza-attributable outcomes Although ILI is the usual surveillance case definition of influenza, less than $30 \%$ of ILI during influenza season is caused by influenza [23], which may additionally present as other respiratory [24-26] and non-respiratory conditions [5, 27]. Consequently, influenza infections are rarely suspected or laboratory-confirmed, making direct measurement of infection difficult. As the circulation of influenza is minimal outside the influenza season, the burden of influenza can instead be estimated by comparing health outcomes during influenza 
season to those during the off-season [12]. However, this approach may be confounded by cyclic trends, since respiratory outcomes rise and fall each year even in the absence of significant influenza activity [27-29]). Researchers have accounted for cyclic trends and other potential confounders by using statistical models to estimate the outcomes that would have occurred, during influenza season, in the absence of circulating influenza. This counterfactual is then compared with estimated outcomes under actual conditions, to obtain an influenza-attributable estimate of outcome frequency [2-5, 27].

We modelled rates of each outcome in adults with and without diabetes using unconditional time-varying Poisson regression. Person-time was split into weeks, with follow-up time in person-years modelled as an offset term. A binary indicator for influenza season (i.e. influenza season vs offseason time) represented the adjusted relative effects of influenza on outcome rates, that is, the 'influenza-attributable effect'. Variables indicating each month were included to account for cyclic trends. A term indicating diabetes status was included to adjust for the increased likelihood of hospitalisation or presentation for medical attention among adults with diabetes generally, regardless of influenza susceptibility or severity. The interaction of diabetes status and influenza-season terms provided a formal test of differences in relative rates of influenza-attributable illness among those with and without diabetes. An example would be if influenza season was associated with $10 \%$ (RR 1.10) and $20 \%$ (RR 1.20) relative increases in the rates of ALL in non-diabetic and diabetic adults, respectively. Given a statistically significant interaction term (i.e. interaction RR 1.20/1.10=1.09), we could infer that influenza increases ALL to a greater extent in those with diabetes, and that those with diabetes are therefore more susceptible to influenza. Greater rates of influenzaattributable outcomes may also indicate greater severity of influenza if outcomes considered more serious (i.e. PI, ALL) are disproportionately increased compared with outcomes capturing less serious healthcare use (i.e. ILI, which includes both physician visits and hospitalisations).

All models were additionally adjusted for age, sex, location of residence, socioeconomic status (SES), comorbidities, number of physician visits in the previous year, receipt of current influenza vaccination for the influenza year, previous receipt of pneumococcal vaccination and secular (i.e. temporal) trends. SES was based on the census-derived income quintile of each subject's postal code area of residence [30, 31]. Comorbidity was represented by the number of major aggregate diagnostic groups (ADGs) accrued during the previous 2 years (i.e. 0,1 , or $\geq 2$ major ADGs; major $A D G s$ are listed in the footnote to Table 1) [32, 33]. Influenza vaccination is an important confounder because it is felt to reduce the sequelae of influenza in those with diabetes. Since the effect of influenza vaccination is difficult to disentangle from a tendency for those with greater health status to be vaccinated [15], adjusting for influenza vaccination has the additional benefit of reducing confounding from unmeasured differences in health status. Finally, we adjusted for secular, in addition to cyclic, trends using indicator variables for each year $[3,5]$. All variables were updated every $1 \mathrm{July}$, except for vaccination status, which was updated after the week of receipt. To check for overdispersion, we performed the same analysis using negative binomial regression and the results were virtually identical (data not shown). Results were considered statistically significant at a standard of $p<0.05$. Analyses were performed using SAS 9.2 (SAS Institute, Cary, NC, USA).

\section{Results}

We identified 60,118 working-age adults with diabetes in Manitoba from 2000 to 2008. Of these, 58,577 adults (97\%) were matched to one or more non-diabetic control subjects. Our study included 56,513 adults with diabetes and 110,202 non-diabetic controls with complete data, who together contributed 745,777 person-years of follow-up. Adults with and without diabetes were similar in age and sex. At cohort entry, those with diabetes had a median age of 51 years (interquartile range [IQR] 15), those without diabetes had a median age of 50 (IQR 15); $51-52 \%$ of subjects were men in both groups. Patients with diabetes were more likely to have a belowmedian income, made more physician visits, had greater comorbidity based on major ADGs and were more likely to have been vaccinated against influenza or pneumococcal infection, compared with non-diabetic controls $(p<0.05)$ (Table 1). On average, our study included 31,139 working-age adults with diabetes each year, representing $58 \%$ of diabetic adults of any age, and approximately $3 \%$ of the entire Manitoba population.

During the follow-up period, we observed 251,144 ILIs, 1,892 PIs, and 36,955 ALLs. Actual and model-based counts of each outcome, including model-estimated counts in the counterfactual absence of influenza season, are shown in Fig. 1. Outcomes demonstrated a cyclical rise and fall each year. Where distinguishable, excess outcomes during influenza season are shown.

In adults with diabetes, influenza season was associated with statistically significant increases in the rates of all outcomes studied after adjusting for cyclic trends and other potential confounders (Table 2). In those without diabetes, influenza season was associated with increased ILI. The relative effect of influenza appeared to be greater in diabetic adults than in non-diabetic adults for PI (RR 1.35 vs 1.13 [diabetes vs no diabetes] for the effect of influenza) and ALL (RR 1.06 vs 1.00 [diabetes vs no diabetes] for the effect of influenza) (Table 2). While the former difference was not statistically significant $(p=0.107)$, our formal test of interaction showed 
Table 1 Cohort characteristics

\begin{tabular}{|c|c|c|c|c|}
\hline \multirow[t]{2}{*}{ Variable } & \multicolumn{2}{|c|}{$\begin{array}{l}\text { Diabetes } \\
\text { Age, years (median, IQR): 51, } 15\end{array}$} & \multicolumn{2}{|c|}{$\begin{array}{l}\text { No diabetes } \\
\text { Age, years (median, IQR): 50, } 15\end{array}$} \\
\hline & No. of subjects & Proportion & No. of subjects & Proportion \\
\hline \multicolumn{5}{|l|}{ Sex } \\
\hline Male & 29,953 & 0.53 & 57,738 & 0.52 \\
\hline Female & 26,560 & 0.47 & 52,464 & 0.48 \\
\hline \multicolumn{5}{|c|}{ Income quintile } \\
\hline Upper & 25,666 & 0.45 & 61,809 & 0.56 \\
\hline Lower & 30,847 & 0.55 & 48,393 & 0.44 \\
\hline \multicolumn{5}{|l|}{ Residence } \\
\hline Urban & 33,363 & 0.59 & 65,702 & 0.60 \\
\hline Rural & 23,150 & 0.41 & 44,500 & 0.40 \\
\hline \multicolumn{5}{|c|}{ No. of medical visits - previous year } \\
\hline 0 & 32,516 & 0.58 & 79,948 & 0.73 \\
\hline $1-2$ & 14,927 & 0.26 & 22,526 & 0.20 \\
\hline 3 or more & 9,070 & 0.16 & 7,728 & 0.07 \\
\hline \multicolumn{5}{|c|}{ No. of major ADGs ${ }^{\mathrm{a}}$} \\
\hline 0 & 24,642 & 0.44 & 68,649 & 0.62 \\
\hline 1 & 16,807 & 0.30 & 28,734 & 0.26 \\
\hline 2 or more & 15,064 & 0.27 & 12,819 & 0.12 \\
\hline \multicolumn{5}{|c|}{ Influenza vaccination - previous year } \\
\hline Yes & 8,813 & 0.16 & 7,842 & 0.07 \\
\hline No & 47,700 & 0.84 & 102,360 & 0.93 \\
\hline \multicolumn{5}{|c|}{ Pneumococcal vaccination (any) } \\
\hline Yes & 2,497 & 0.04 & 2,128 & 0.02 \\
\hline No & 54,016 & 0.96 & 108,074 & 0.98 \\
\hline
\end{tabular}

Table enumerates subjects at cohort entry. Proportions vary if subjects are enumerated over their follow-up periods on 1 July each year, with person-years of follow-up as the denominator (e.g. greater prevalence of influenza vaccinations), but relationships among those with vs those without diabetes remain similar ${ }^{a}$ Number of major ADGs over the previous 2 years: ADG3 (time limited: major), ADG4 (time limited: major-primary infections), ADG9 (likely to recur: progressive), ADG11 (chronic medical: unstable), ADG16 (chronic specialty: unstable — orthopaedic), ADG22 (injuries/adverse effects: major), ADG25 (psychosocial: recurrent or persistent, unstable) and ADG32 (malignancy)

All between group differences, $p<0.05$ on Wilcoxon rank-sum or $\chi^{2}$ tests

statistical significance for the $6 \%$ greater relative increase in ALL due to influenza in diabetic adults $(p=0.044)$.

In diabetic adults, influenza contributed $13 \%, 26 \%$ and $6 \%$ of ILIs, PIs and ALLs occurring during influenza season, respectively (Table 2). For ALLs, virtually all of these influenzaattributable outcomes (i.e. 54/55 events [98\%]) were due to the increased effects of influenza in diabetic adults (Table 3). In absolute terms, influenza season was associated with an additional six ALLs per one-thousand diabetic person-years.

\section{Discussion}

The burden of influenza is difficult to estimate due to lack of specific outcome measures in administrative data. Using statistical regression techniques, we have distinguished the effects of seasonal influenza in working-age Manitoba adults with and without diabetes. Influenza was associated with increased rates of physician visits and hospitalisations for influenza-like illness in non-diabetic adults, accounting for $15 \%$ of ILI during influenza season. In diabetic adults, influenza was associated with increased ILI, PI and ALL, accounting for $13 \%, 26 \%$ and $6 \%$ of these outcomes, respectively. Compared with working-age non-diabetic adults, workingage adults with diabetes experienced a significantly greater $6 \%$ increase in influenza-attributable ALLs. Since a similar difference was not observed for ILI, we infer that adults with diabetes experience a disproportionately greater susceptibility to more serious manifestations of influenza, requiring hospitalisation. 
Fig. 1 Actual vs projected outcomes in working-age adults with and without diabetes. Outcome numbers in adults without diabetes were estimated for a 2:1 matched group of controls and therefore do not represent actual numbers for adults in Manitoba without diabetes. + symbol represents weekly numbers of each outcome. Grey bars, influenza season; dotted line, 1 July for the influenza season indicated; green line, projected number of events; blue line, projected number of events in the absence of circulating influenza; red line, number of influenza-attributable events per week (i.e. green line minus blue line)

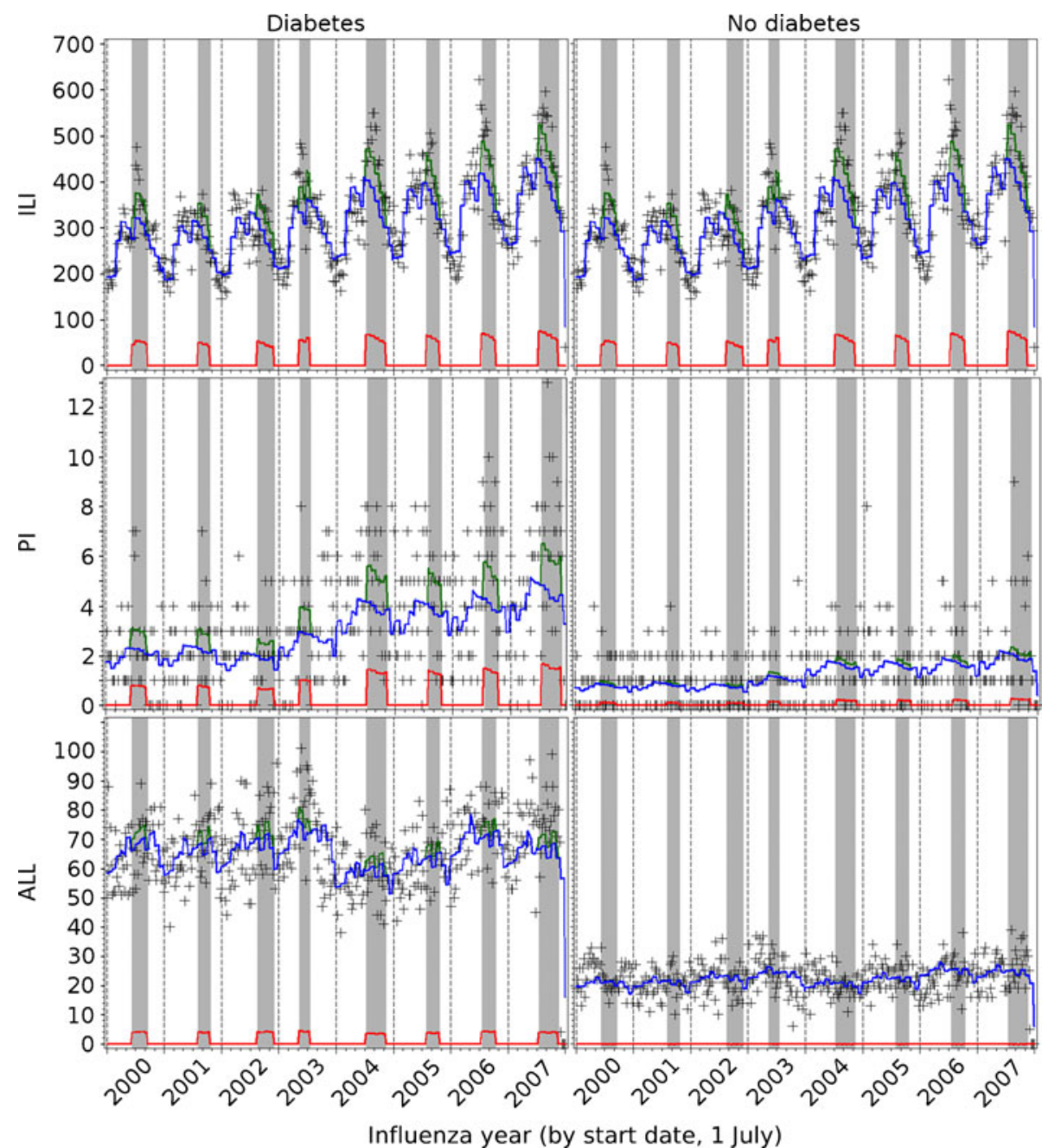

In absolute terms, working-age adults with diabetes experienced an additional six hospitalisations due to influenza per 1,000 adults. Notably, if these same adults had not had diabetes they would not have experienced any ALLs due to influenza, since influenza did not appear appreciably to increase ALLs in those without diabetes.
Limitations and strengths Our study has several limitations. First, because we relied on a community-level indicator for influenza, ecological bias may arise if outcomes attributed to influenza did not actually occur in patients infected with influenza. For example, respiratory syncytial virus (RSV) co-circulates with influenza and causes similar illness [3].

Table 2 Relative effect of circulating influenza on the incidence of ILI, PI and ALL in adults with and without diabetes

\begin{tabular}{|c|c|c|c|c|c|c|c|c|c|}
\hline \multirow[t]{2}{*}{ Outcome } & \multicolumn{4}{|c|}{ Adults without diabetes } & \multicolumn{4}{|c|}{ Adults with diabetes } & \multirow[b]{2}{*}{ Interaction $\mathrm{p}$ value } \\
\hline & Rate ratio ${ }^{\mathrm{a}}$ & $95 \% \mathrm{CI}$ & $f^{\mathrm{b}}$ & $p$ value & Rate ratio ${ }^{a}$ & $95 \% \mathrm{CI}$ & $f^{\mathrm{b}}$ & $p$ value & \\
\hline ILI & 1.17 & $1.15,1.18$ & 14.5 & $<0.001 * * *$ & 1.15 & $1.13,1.17$ & 13.0 & $<0.001^{* * *}$ & 0.057 \\
\hline PI & 1.13 & $0.91,1.39$ & 11.5 & 0.265 & 1.35 & $1.16,1.56$ & 25.9 & $<0.001 * * *$ & 0.107 \\
\hline ALL & 1.00 & $0.95,1.06$ & 0.3 & 0.889 & 1.06 & $1.02,1.10$ & 5.7 & $0.001 * * *$ & $0.044 *$ \\
\hline
\end{tabular}

Estimates are adjusted for potential confounders (see Methods)

${ }^{a}$ Relative effect of circulating influenza (influenza season vs off-season)

${ }^{\mathrm{b}}$ Percentage of outcomes due to influenza during influenza season: $f=[(\mathrm{RR}-1) / \mathrm{RR}] \times 100 \%$

${ }^{\mathrm{c}}$ The interaction term is defined in the model as the ratio of the two previous risk ratios (RRs), representing the change in the effect of influenza by diabetes status

${ }^{*} p \leq 0.05 ; * * * p \leq 0.001$ 
Table 3 Model-projected numbers of influenza-attributable outcomes during influenza season in adults with and without diabetes

\begin{tabular}{|c|c|c|c|c|}
\hline \multirow[t]{2}{*}{ Outcome } & \multicolumn{3}{|l|}{ Adults with diabetes } & \multirow{2}{*}{$\begin{array}{l}\text { Adults without diabetes } \\
\text { (2:1 matched controls) } \\
\text { Under actual } \\
\text { conditions (projected) }\end{array}$} \\
\hline & $\begin{array}{l}\text { Under actual } \\
\text { conditions (projected) }\end{array}$ & $\begin{array}{l}\text { If subjects did not } \\
\text { have diabetes } \\
\text { (counterfactual) }\end{array}$ & $\begin{array}{l}\text { Difference }^{\mathrm{a}} \text { (additional } \\
\text { influenza-attributable } \\
\text { outcomes due to having diabetes) }\end{array}$ & \\
\hline \multicolumn{5}{|l|}{ ILI } \\
\hline$n$ (average per year) & 627 & 589 & 38 & 794 \\
\hline No. of persons (average per year) & 8,786 & 8,786 & 8,786 & 16,202 \\
\hline Rate (/1,000 person-years) & 71.38 & 67.06 & 4.32 & 49.01 \\
\hline \multicolumn{5}{|l|}{ PI } \\
\hline$n$ (average per year) & 16 & 2 & 13 & 2 \\
\hline No. of persons (average per year) & 8,786 & 8,786 & 8,786 & 16,202 \\
\hline Rate (/1,000 person-years) & 1.77 & 0.28 & 1.50 & 0.14 \\
\hline \multicolumn{5}{|l|}{ ALL } \\
\hline$n$ (average per year) & 55 & 1 & 54 & 1 \\
\hline No. of persons (average per year) & 8,786 & 8,786 & 8,786 & 16,202 \\
\hline Rate (/1,000 person-years) & 6.24 & 0.12 & 6.12 & 0.07 \\
\hline
\end{tabular}

Counts and rates for adults without diabetes were estimated for the matched comparison group of non-diabetic subjects, and therefore do not represent the actual numbers of influenza-attributable outcomes in adults in Manitoba without diabetes

a 'Projected' minus 'counterfactual'

Unaccounted RSV would lead to overestimates of influenzaattributable illness. Because the proportion of unaccounted RSV is not likely to be different in those with diabetes, our estimates of the relative effects of diabetes on influenzaattributable illness may not be affected. Indeed, we obtained similar results using a more specific measure of influenza activity - the proportion of respiratory specimens positive for influenza virus (data not shown).

Second, as the present study was observational, other unrecognised confounders may affect our findings. For example, obesity has emerged as a risk factor for hospitalisation and severe outcomes in pH1N1 cases [34-36]. It remains unclear from the $\mathrm{pH} 1 \mathrm{~N} 1$ literature whether obesity is an independent risk factor for influenza or whether the association between obesity and severe outcomes of influenza is itself confounded by diabetes, cardiovascular disease and other obesity-related comorbidities [36]. One recent study of the NHANES cohort found a consistent association between obesity and pH1N1 hospitalisations/death, after stratifying by comorbidity, suggesting a true diathesis [34]. In the seasonal influenza literature, Kwong et al have demonstrated an association between obesity and increased respiratory hospitalisations, after adjusting for diabetes and cardiovascular disease [37]. Obesity may therefore account for the association between diabetes and influenzaattributable illness to an unknown extent [38]. Other clinical variables we were unable to include were the specific type of diabetes; and variables related to diabetes severity and control, such as $\mathrm{HbA}_{1 \mathrm{c}}$. The incorporation of such variables in future studies would provide additional evidence helping to confirm or disprove a diabetes-specific effect. Our results likely generalise to those with type 2 diabetes, which comprises the vast majority of diabetes in adults. We adjusted for comorbidity using the number of ADGs instead of specific individual-level diagnoses, due to practical modelling limitations.

Finally, our data is limited to healthcare use, which may under-represent the total burden of influenza. Many workingage adults with influenza do not present for medical attention although they may still incur absenteeism and productivity losses [1, 39]. Previous studies have suggested that patients with diabetes who experience infectious diseases may be more likely to present for medical attention and to be hospitalised [40, 41], and this may lead to overestimates of influenza-attributable illness specifically in diabetic adults. Because increased medical attention likely applies generally, regardless of infectious aetiology (e.g. ILI, PI or ALL of both influenza and noninfluenza aetiologies), we have accounted for this source of potential confounding by singling out differences in the effects of circulating influenza among diabetic and non-diabetic adults, while adjusting analytically for the effects of diabetes alone.

Three previous studies have compared influenzaattributable outcomes in patients with and without diabetes during periods of seasonal influenza. Schanzer et al noted increased influenza-attributable primary respiratory admissions in patients with diabetes compared with those without diabetes, but did not report diabetes-specific risk estimates [13]. Bouter et al found that diabetes increased the risk of both pneumonia hospitalisations and subsequent mortality to a greater extent during years with significant influenza activity 
[11]. Finally, Neuzil et al reported a fivefold (unadjusted) higher rate of influenza-attributable cardiopulmonary hospitalisations or deaths in working-age women with diabetes [12]. The limitations of these studies include potential bias from the use of hospital-based comparison groups [11], lack of adjustment for comorbidities and vaccination status [11-13] and inadequate adjustment for seasonality [12], as mentioned earlier. Additionally, several studies have examined the effects of diabetes on pH1N1 influenza. Diabetes appears disproportionately represented in most studies of confirmed pH1N1 cases requiring hospitalisation [36, 42-45]. Some pH1N1 studies have also identified diabetes as a risk factor for severe outcomes following hospitalisation [45], although other studies have not $[46,47]$. Whether the high prevalence of diabetes in $\mathrm{pH} 1 \mathrm{~N} 1$ studies can be attributed to a diabetes-effect on risk [45], confounding due to other comorbidities [46, 47] or due to adults with diabetes being more readily admitted on a precautionary basis [35] remains unclear. Moreover, since over one-third of diabetes is undiagnosed in the community [48], the lack of a population control group in case series of pH1N1 hospitalisations makes it difficult to ascertain whether the apparent effect of diabetes on $\mathrm{pH} 1 \mathrm{~N} 1$ hospitalisations is due to increased detection of diabetes in tertiary care. The prevalence of diabetes in $\mathrm{pH} 1 \mathrm{~N} 1$ patients may be consistent with its community prevalence if undiagnosed diabetes is included [46].

To our knowledge, our study is one of only two studies [12] to have identified and followed individuals, in the general population, with and without diabetes, for influenzaattributable outcomes. The use of administrative databases ensures comparable data quality among all study subjects. Our study used a validated case definition [16] to define diabetes status before outcome ascertainment, thus avoiding differential detection of undiagnosed diabetes in adults hospitalised for a study outcome. Moreover, ours is the only study to have adjusted for comorbidities, vaccination status and cyclic trends apart from influenza. Insofar as diabetes was found to be a risk factor for influenza-attributable hospitalisations, our results agree with those of previous seasonal influenza studies [11-13] as well as those of certain pH1N1 studies [45]. In contrast to previous studies, the effect we demonstrated was much smaller and subtler in magnitude. These findings highlight the importance of appropriate adjustment for potential confounders and cyclic trends, which may otherwise lead the burden of seasonal influenza to be overestimated in high-risk populations. Our findings also suggest that we should be cautious of $\mathrm{pH} 1 \mathrm{~N} 1$ series showing a high prevalence of diabetes, although our data did not include 2009 and are therefore not straightforwardly applicable.

Clinical and policy implications Our results suggest that working adults with diabetes experience greater risk of influenza-related ALLs. The public health impact of diabetes on the burden of influenza in Manitoba may be summarised as an additional six hospitalisations per 1,000 diabetic personyears. A previous economic analysis of a universal vaccination programme in Ontario estimated the cost of a pneumoniaor influenza-related hospitalisation to be $\$ 6,418$ Canadian dollars (CAD), and the cost of a universal vaccination programme to be $\$ 7.55 \mathrm{CAD}$ per dose delivered [49]. Using these figures as rough approximations, it may be cost-saving to target working-age adults with diabetes for vaccination, with a presumed rate of vaccine effectiveness as low as $20 \%$. Of course, the unit cost of a diabetes-specific vaccination programme may be much higher, and the actual effectiveness of influenza vaccinations in high-risk populations is not well established. While observational studies suggest that vaccination against influenza reduces hospitalisations in working-age adults with diabetes $[10,50]$, these benefits may be attributable to healthy user bias [15]. Our observation that workingage adults with diabetes experience a greater burden of influenza than similar non-diabetic adults provides a clinical justification for targeted anti-influenza interventions; identifying particular interventions and evaluating their effectiveness in this population are questions for further research.

Vaccination guidelines indirectly single out working-age adults with diabetes for routine vaccination. We have demonstrated an increased burden of influenza in this population. Randomised trials are needed to confirm actual vaccine effectiveness in this group. Formal economic studies are also required, to ascertain the extent to which identifying diabetes as a high-risk indication for vaccination may mitigate the healthcare use and costs associated with influenza. Until such studies are available, our work represents the strongest current evidence highlighting the burden of influenza, and the potential benefits of influenza vaccination, in diabetic adults.

Acknowledgements The authors would like to acknowledge the support and expertise of C. Burchill (Manitoba Centre for Health Policy, Winnipeg, MB, Canada), who facilitated data access and analyses. This study was approved by the Health Information Privacy Committee of Manitoba (2011/2012-16). The results and conclusions presented are those of the authors. No official endorsement by Manitoba Health is intended or should be inferred.

Funding This work was supported by an open operating grant from the Canadian Institutes of Health Research (MOP-119316). DL is funded by $\mathrm{MD} / \mathrm{PhD}$ studentships from CIHR and Alberta Innovates-Health Solutions (AIHS). DTE holds a CIHR New Investigator award, and is an AIHS Population Health Investigator. SRM holds the Endowed Chair in Patient Health Management, supported by the Faculty of Medicine and Dentistry and the Faculty of Pharmacy and Pharmaceutical Sciences, University of Alberta, and is an AIHS Health Scholar. JAJ is an AIHS Senior Scholar and a Centennial Professor at the University of Alberta.

Duality of interest The authors declare that there is no duality of interest associated with this manuscript. 
Contribution statement JAJ had full access to all the data in the study and takes responsibility for the integrity of the data and accuracy of the data analysis. All authors contributed to the design and conception of the study, and the interpretation of data. DL performed the data analysis, and wrote the first draft of the article. DL, DTE, SRM, AK and JAJ contributed to subsequent revisions. All authors reviewed and approved the final draft.

\section{References}

1. Molinari NAM, Ortega-Sanchez IR, Messonnier ML et al (2007) The annual impact of seasonal influenza in the US: measuring disease burden and costs. Vaccine 25:5086-5096

2. Thompson WW, Shay DK, Weintraub E et al (2004) Influenza-associated hospitalizations in the United States. JAMA 292:1333-1340

3. Schanzer DL, Langley JM, Tam TWS (2008) Role of influenza and other respiratory viruses in admissions of adults to Canadian hospitals. Influenza Other Respir Viruses 2:1-8

4. Thompson WW, Shay DK, Weintraub E et al (2003) Mortality associated with influenza and respiratory syncytial virus in the United States. JAMA 289:179-186

5. Schanzer DL, Tam TWS, Langley JM, Winchester BT (2007) Influenza-attributable deaths, Canada 1990-1999. Epidemiol Infect 135:1109-1116

6. Clinical Practice Guidelines Expert Committee (2008) Influenza and pneumococcal immunizations. Can J Diabetes 32:S86-S87

7. American Diabetes Association (2009) Standards of medical care in diabetes-2009. Diabetes Care 32:S13-S61

8. National Advisory Committee on Immunizations (2011) Statement on seasonal influenza vaccine for 2011-2012. Can Commun Dis Rep 37:1-55

9. Advisory Committee on Immunization Practices (2010) Prevention and control of influenza with vaccines. MMWR Morb Mortal Wkly Rep $59: 1-62$

10. Smith SA, Poland GA (2000) Use of influenza and pneumococcal vaccines in people with diabetes. Diabetes Care 23:95-108

11. Bouter KP, Diepersloot RJA, van Romunde LKJ et al (1991) Effect of epidemic influenza on ketoacidosis, pneumonia and death in diabetes mellitus: a hospital register survey of 1976-1979 in the Netherlands. Diabetes Res Clin Pract 12:61-68

12. Neuzil KM, Reed GW, Mitchel EF Jr, Griffin MR (1999) Influenzaassociated morbidity and mortality in young and middle-aged women. JAMA 281:901-907

13. Schanzer DL, Langley JM, Tam TWS (2008) Co-morbidities associated with influenza-attributed mortality, 1994-2000, Canada. Vaccine 26:4697-4703

14. Health Canada (2011) Canada Health Act Annual Report 2010-2011. Health Canada, Ottawa, ON. Available from www.hc-sc.gc.ca/hcssss/pubs/cha-lcs/2011-cha-lcs-ar-ra/index-eng.php, accessed 24 June 2011

15. Lau D, Eurich DT, Majumdar SR, Katz A, Johnson JA (2013) Effectiveness of influenza vaccination in working-age adults with diabetes: a population-based cohort study. Thorax 68:658-663

16. Hux JE, Ivis F, Flintoft V, Bica A (2002) Diabetes in Ontario: determination of prevalence and incidence using a validated administrative data algorithm. Diabetes Care 25:512-516

17. Hottes TS, Skowronski DM, Hiebert B et al (2011) Influenza vaccine effectiveness in the elderly based on administrative databases: change in immunization habit as a marker for bias. PLoS ONE 6:e22618

18. Jackson LA, Jackson ML, Nelson JC, Neuzil KM, Weiss NS (2006) Evidence of bias in estimates of influenza vaccine effectiveness in seniors. Int J Epidemiol 35:337-344
19. Eurich DT, Marrie TJ, Johnstone J, Majumdar SR (2008) Mortality reduction with influenza vaccine in patients with pneumonia outside 'flu' season: pleiotropic benefits or residual confounding? Am J Resp Crit Care Med 178:527-533

20. Public Health Agency of Canada (2009) Fluwatch - definitions for the 2009-2010 season. Public Health Agency of Canada, Ottawa

21. Tsui FC, Wagner MM, Dato V, Chang CC (2001) Value of ICD-9 coded chief complaints for detection of epidemics. Proc AMIA Symp, pp 711-715

22. Belongia E, Irving S, Waring S et al (2010) Clinical characteristics and 30-day outcomes for influenza a 2009 (H1N1), 2008-2009 (H1N1), and 2007-2008 (H3N2) infections. JAMA 304:1091-1098

23. Ebell MH (2005) Diagnosing and treating patients with suspected influenza. Am Fam Physician 72:1789-1792

24. Louie JK, Hacker JK, Gonzales R et al (2005) Characterization of viral agents causing acute respiratory infection in a San Francisco University Medical Center Clinic during the influenza season. Clin Infect Dis 41:822-828

25. Heikkinen T, Järvinen A (2003) The common cold. Lancet 361:51-59

26. Johnstone J, Majumdar S, Fox J, Marrie T (2008) Viral infection in adults hospitalized with community-acquired pneumonia: prevalence, pathogens, and presentation. Chest 134:1141-1148

27. Alling DW, Blackwelder WC, Stuart-Harris CH (1981) A study of excess mortality during influenza epidemics in the United States, 1968-1976. Am J Epidemiol 113:30-43

28. Collins SD (1930) Influenza-pneumonia mortality in a group of about 95 cities in the United States, 1920-1929. Public Health Rep 45:361-406

29. Serfling RE (1963) Methods for current statistical analysis of excess pneumonia-influenza deaths. Public Health Rep 78:494-506

30. Alter DA, Naylor CD, Austin P, Tu JV (1999) Effects of socioeconomic status on access to invasive cardiac procedures and on mortality after acute myocardial infarction. N Engl J Med $341: 1359-1367$

31. Krieger N (1992) Overcoming the absence of socioeconomic data in medical records: validation and application of a census-based methodology. Am J Public Health 82:703-710

32. Reid RJ, MacWilliam L, Verhulst L, Roos N, Atkinson M (2001) Performance of the ACG case-mix system in two Canadian provinces. Med Care 39:86-99

33. Hilderman T, Katz A, Derksen S et al (2011) Manitoba immunization study. Manitoba Centre for Health Policy, Winnipeg

34. Morgan OW, Bramley A, Fowlkes A et al (2010) Morbid obesity as a risk factor for hospitalization and death due to 2009 pandemic influenza A(H1N1) disease. PLoS ONE 5:e9694

35. Ganatra RB, McKenna JJ, Bramley AM et al (2013) Adults with diabetes hospitalized with pandemic influenza A(H1N1)pdm09-U.S. 2009. Diabetes Care 36:e94

36. Van Kerkhove MD, Vandemaele KAH, Shinde V et al (2011) Risk factors for severe outcomes following 2009 influenza A (H1N1) infection: a global pooled analysis. PLoS Med 8:e1001053

37. Kwong JC, Campitelli MA, Rosella LC (2011) Obesity and respiratory hospitalizations during influenza seasons in Ontario, Canada: a cohort study. Clin Infect Dis 53:413-421

38. Jain S, Chaves SS (2011) Obesity and influenza. Clin Infect Dis 53:422-424

39. Reed C, Angulo FJ, Swerdlow DL et al (2009) Estimates of the prevalence of pandemic (H1N1) 2009, United States, April-July 2009. Emerg Infect Dis 15:2004-2007

40. Shah BR, Hux JE (2003) Quantifying the risk of infectious diseases for people with diabetes. Diabetes Care 26:510-513

41. Jackson ML, Neuzil KM, Thompson WW et al (2004) The burden of community-acquired pneumonia in seniors: results of a populationbased study. Clin Infect Dis 39:1642-1650 
42. Louie JK, Acosta M, Winter K et al (2009) Factors associated with death or hospitalization due to pandemic 2009 influenza A(H1N1) infection in California. JAMA 302:1896-1902

43. Jain S, Kamimoto L, Bramley AM et al (2009) Hospitalized patients with 2009 H1N1 influenza in the United States, April-June 2009. N Engl J Med 361:1935-1944

44. Kumar A, Zarychanski R, Pinto R et al (2009) Critically ill patients with 2009 influenza $\mathrm{A}(\mathrm{H} 1 \mathrm{~N} 1)$ infection in Canada. JAMA 302:1872-1879

45. Allard R, Leclerc P, Tremblay C, Tannenbaum T-N (2010) Diabetes and the severity of pandemic influenza A (H1N1) infection. Diabetes Care 33:1491-1493

46. Jiménez-García R, Hernández-Barrera V, Rodríguez-Rieiro C et al (2013) Hospitalizations from pandemic influenza [A(H1N1)pdm09] infections among type 1 and 2 diabetes patients in Spain. Influenza Other Respir Viruses 7:439-447
47. Cortes Garcia M, Sierra Moros MJ, Santa-Olalla Peralta P, Hernandez-Barrera V, Jimenez-Garcia R, Pachon I (2012) Clinical characteristics and outcomes of diabetic patients who were hospitalised with 2009 pandemic influenza A H1N1 infection. J Infect 64:218-224

48. Cowie CC, Rust KF, Byrd-Holt DD et al (2006) Prevalence of diabetes and impaired fasting glucose in adults in the U.S. population: National Health And Nutrition Examination Survey 19992002. Diabetes Care 29:1263-1268

49. Sander B, Kwong JC, Bauch CT et al (2010) Economic appraisal of Ontario's Universal Influenza Immunization Program: a cost-utility analysis. PLoS Med 7:e1000256

50. Looijmans-Van Den Akker I, Verheij TJM, Buskens E, Nichol KL, Rutten GEHM, Hak E (2006) Clinical effectiveness of first and repeat influenza vaccination in adult and elderly diabetic patients. Diabetes Care 29:1771-1776 